\title{
The effect of fishing on hysteresis in Caribbean coral reefs
}

\author{
Julie C. Blackwood • Alan Hastings • Peter J. Mumby
}

Received: 23 March 2010 / Accepted: 19 October 2010 / Published online: 13 November 2010

(C) The Author(s) 2010. This article is published with open access at Springerlink.com

\begin{abstract}
Coral resilience is important for withstanding ecological disturbances as well as anthropogenic changes to the environment. However, the last several decades have demonstrated a decline in resilience that has often resulted in phase shifts to a degraded coraldepleted state with high levels of algal abundance. A major defining issue in current research is to identify when and how it is possible to reverse these phase shifts allowing for the ecosystem to escape coral depletion and maintain coral-based ecosystem services. We extend an analytic model to focus on the effects of overharvesting of herbivorous reef fish in the Caribbean by explicitly including grazer dynamics which introduces feedbacks between habitat and grazer abundance
\end{abstract}

Electronic supplementary material The online version of this article (doi:10.1007/s12080-010-0102-0) contains supplementary material, which is available to authorized users.

J. C. Blackwood

Department of Ecology and Evolutionary Biology,

University of Michigan, Ann Arbor, MI 48109, USA

J. C. Blackwood $(\varangle)$

Center for the Study of Complex Systems,

University of Michigan, Ann Arbor, MI 48109, USA

e-mail: juliecb@umich.edu

\author{
A. Hastings \\ Department of Environmental Science and Policy, \\ University of California-Davis, Davis, CA 95616, USA \\ e-mail: amhastings@ucdavis.edu \\ P. J. Mumby \\ School of Biological Sciences \& ARC Centre of Excellence \\ for Coral Reef Studies, University of Queensland, St. Lucia, \\ Brisbane, Qld 4072, Australia \\ e-mail: p.j.mumby@uq.edu.au
}

posing constraints on management options excluded in previous studies. This allows us to develop ecosystembased management recommendations for two distinct scenarios of coral reef recovery: The first follows significant habitat damage in response to a large disturbance and the second maintains reef structure but has suffered from events such as coral bleaching. We identify critical fishing effort levels to allow for coral recovery and demonstrate that regions exhibiting severe damage to reef structure have little resilience implying that fishing reductions should be coupled with other restoration methods. Regions that are coraldepleted but maintain reef structure allow for recovery given sufficiently small levels of fishing mortality. However, we demonstrate the difference in recovery time in response to varying levels of control efforts on fishing.

Keywords Coral reefs • Resilience • Phase shifts • Hysteresis

\section{Introduction}

In a biological system with multiple stable states, hysteresis occurs when the state of the system depends strongly on its previous history. In other words, the critical condition under which the system switches from one stable state to another is different from the condition that will allow the system to return to the original state (Holling 1973). This phenomenon has been shown to appear in both aquatic and terrestrial systems, a classic example being lake ecosystems in which nutrient loading may lead to algal blooms but returning to the previous state requires a more substantial 
decrease in nutrients (see review in Scheffer et al. 2001). Another important example lies in deserts where desertification and abundant vegetation exist as alternative stable states, a result of feedbacks between soil and plant abundance (von de Koppel et al. 1997). However, despite emphasis on the importance of hysteresis and resilience (the ability to return to a particular state following a perturbation) in theoretical investigations, there has been a much more limited study of the phenomenon in specific systems to justify its importance in understanding and implementing management options. Here, we use ideas from hysteresis and an analytic model to guide ecosystem-based management options for maintaining the resilience of a coral reef ecosystem.

Coral reefs, particularly those in the species-poor Caribbean, are widely thought to exhibit at least two alternate stable states (Knowlton 1992; Bellwood et al. 2004). One such state corresponds to high levels of coral cover, and the other less desirable state corresponds to coral depletion. Unfortunately, many of the ecosystem services provided by these systems such as fisheries and coastal defense from hurricanes (Moberg and Folke 1999) are founded on the corals themselves (Done et al. 1996). Thus, the formation of a stable coral-depleted state that is usually associated with relatively high levels of macroalgae (seaweed) is considered undesirable from a human perspective.

To reduce the occurrence of such coral-depleted states, it is important that systems have the resilience required to withstand natural ecological fluctuations such as those driven by hurricanes (Bythell et al. 2000) and anthropogenic disturbance such as eutrophication, fishing, and global warming (Hoegh-Guldberg et al. 2007). However, whether sufficient resilience exists to combat such stressors is questionable for many reef ecosystems and the Caribbean in particular. For example, in an analysis of recovery trajectories, Connell found limited evidence of recovery taking place in Caribbean ecosystems (Connell 1997). One possible reason for this lack of recovery is the diseaseinduced depletion of the herbivorous urchin Diadema antillarum in 1983 which rapidly reduced the natural controls on algal populations (Hughes 1994). Today, large-bodied parrotfish such as the stoplight parrotfish (Sparisoma viride) remain the predominant grazers of algae on the vast majority of Caribbean reefs, though limited recovery has occurred in shallow reefs (Carpenter and Edmunds 2006). Given that parrotfish have been shown empirically to exert a profound influence on the level of macroalgal cover on contemporary Caribbean reefs (Williams and Polunin 2000;
Mumby et al. 2006), we develop a model of coral reef resilience that explicitly includes the management of parrotfish exploitation.

Mumby et al. (2007) introduced a model with grazing at an imposed level demonstrating that a coral reef ecosystem may lose resilience and shift to a coraldepleted state through reductions in grazing intensity. We extend this model by the explicit inclusion of the population dynamics of the primary grazer (parrotfish) to identify situations that allow for coral recovery through increases in resilience, mostly through a reduction in the fishing of parrotfishes. More specifically, grazing is now implemented as a dynamic variable with dependence on the population dynamics of parrotfish in light of fishing and natural mortality. This introduces important constraints on possible management options that only arise when the grazer dynamics are explicitly included. Consequently, by considering the underlying parrotfish dynamics, we introduce two important feedbacks excluded in our previous work: Parrotfish dynamics affect the likelihood of coral recovery by modifying grazing levels, and coral recovery affects the surrounding habitat which, in turn, impacts parrotfish abundance.

Contrary to our previous work, the model formulation we provide in this paper allows us to directly investigate the effects of various physical disturbances on resilience of coral reefs. Specifically, two scenarios are investigated. In the first, it is assumed that coral habitat is the primary limiting resource for parrotfish. This situation would arise in a system that has been heavily disturbed such that the reef structure is flat and lacking appropriate refugia for parrotfish from predators. A recovery of corals in this situation would add threedimensional structure and enhance parrotfish recruitment (Tolimieri 1998), thereby generating a positive relationship between coral cover and the number of grazers. The second scenario assumes that food availability (i.e., algae) is the primary limiting factor. This scenario represents the effects of coral mortality on a structurally complex reef and may arise as successive coral bleaching events remove living coral tissue. Positive numerical responses of parrotfishes to an increase in food have been documented after a major hurricane and bleaching event killed coral in Belize without reducing the structural complexity of the reef (Mumby et al. 2005). We consider the effects of reductions in fishing effort, identifying critical situations that allow for coral recovery in the face of disturbance. In addition, we demonstrate the analytic difficulty in determining regions that may be controlled via reduced fishing effort to a stable state with high coral cover. 


\section{Methods}

\section{Original model}

We begin with an analytic model developed by Mumby et al. (2007) in terms of the fraction of seabed in a given area and then modify the model to allow for additional features. It was assumed that a particular region of the seabed is covered entirely by macroalgae $(M)$, coral $(C)$, and algal turfs $(T)$ so that $M+C+T=1$ at any given time. The reef dynamics are thus described as a system coupled nonlinear differential equations:

$$
\begin{aligned}
\frac{d M}{d t} & =a M C-\frac{g M}{M+T}+\gamma M T \\
\frac{d C}{d t} & =r T C-d C-a M C \\
\frac{d T}{d t} & =\frac{g M}{M+T}-\gamma M T-r T C+d C
\end{aligned}
$$

where it is assumed that corals recruit to and overgrow algal turfs at a rate $r$, they have a natural mortality rate of $d$, are overgrown by macroalgae at a rate $a$, and macroalgae spread vegetatively over algal turfs at a rate $\gamma$. Space freed by coral mortality is assumed to be recolonized by algal turfs. Additionally, parrotfish graze macroalgae without distinction from algal turfs at a rate $g$ and $\frac{M}{M+T}$ is simply the proportion of grazing that affects macroalgae. It is assumed that grazing crops macroalgae giving rise to algal turfs and grazing on algal turfs simply prevents macroalgal succession from the turf or the vegetative growth of macroalgae across the turf (consequently, no additional term is needed for the grazing of algal turfs). This formulation assumes that the grazing rate, $g$, is the rate at which algae is grazed given the entire region is covered by algae. Thus, algae is targeted by grazers so that if $M+T<$ 1 , the per capita grazing intensity $\left(\frac{g}{M+T}\right)$ is increased. Furthermore, as $M+T \rightarrow 0$, per capita grazing intensity approaches $\infty$ since all grazing is directed at the remaining available algae. An alternative formulation would be to have the grazing term defined by $\frac{g M^{2}}{M+T}$; however, this assumes that grazers do not have searching capabilities.

Only the first two equations of this model are needed, since the fraction of algal turfs is defined by $T=1-M-C$ and consequently $\frac{d T}{d t}$ is simply given by $-\frac{d M}{d t}-\frac{d C}{d t}$. This system is defined on the invariant region given by $0<M+T<1$ and $C, M, T \geq 0$ (see Online Resource 1 for proof and further explanation). The parameterization chosen exhibits similar behavior to a more complex simulation model which had been tested against an independent 20-year data set from Jamaica and was found to follow the observed trends (Mumby et al. 2005). See Table 1 for a full list of parameter values and further explication. This parameterization is appropriate when parrotfish species in the genus Sparisoma dominate the community, such as in Belize (see Online Resource 2 for a sensitivity analysis). Specifically, Sparisoma feed on both macroalgae and algal turfs, and studies from Belize found that each group comprised half of the diet of stoplight parrotfish. In contrast, sites dominated by parrotfish species in the genus Scarus (e.g., Bonaire) feed almost exclusively on algal turfs and therefore have a different impact on the algal community. However, Sparisoma are an important herbivore throughout the vast majority of

\begin{tabular}{|c|c|c|}
\hline Parameter & Value & Definition \\
\hline$z$ & 0.5 & $\begin{array}{l}\text { Dimensionless parameter that determines the strength of the linear relationship between coral cover } \\
\text { and carrying capacity. This value is used but is it shown in the Online Resource } 2 \text { that any feasible } \\
\text { value produces qualitatively similar results. }\end{array}$ \\
\hline$s$ & 0.49 & $\begin{array}{l}\text { Growth rate of parrotfish (year }{ }^{-1} \text { ), estimated using the population doubling time provided in FishBase } \\
\text { (Froese and Paul) under the assumption that fishing effort is absent. }\end{array}$ \\
\hline$a^{\mathrm{a}}$ & 0.1 & Rate macroalgae directly overgrow coral (year $\left.{ }^{-1}\right)$; such limited overgrowth was shown in Lirman (2001). \\
\hline$\gamma^{\mathrm{a}}$ & 0.8 & Rate macroalgae spread vegetatively over algal turfs (year ${ }^{-1}$; Mumby et al. 2005). \\
\hline$r^{\mathrm{a}}$ & 1 & Rate of coral recruitment to algal turfs (year ${ }^{-1}$ ); coral always overgrow algal turfs (Jompa and McCook 2002). \\
\hline$d^{\mathrm{a}}$ & 0.44 & $\begin{array}{l}\text { Natural coral mortality accounts for } 2-4 \% \text { year }^{-1} \text { (Bythell et al. 1993), and predation accounts for } \\
30 \% \text { year }^{-1} \text { (Box and Mumby 2007). The remaining mortality results from coral disease or tissue damage } \\
\text { following bleaching. }\end{array}$ \\
\hline
\end{tabular}
the Caribbean (Floeter et al. 2005).

Table 1 Parameter values and definitions

a The parameters for the original model are those used in Mumby et al., which were based on the parameterization used for the corresponding simulation model (Mumby et al. 2007). Parameter values are appropriately scaled, and brief descriptions of the parameter selection are also included. Since some parameters might be subject to variation (e.g., rate of parrotfish growth), a sensitivity analysis is provided in the Online Resource 2 demonstrating that some variation in these parameters does not affect our qualitative output 
The grazing rate $g$ inherently depends on grazer abundance and is thus subject to variation with changes in available refugia, food abundance, and fishing mortality. To identify the impact of changes in grazing intensity on coral cover, Fig. 1 presents a bifurcation diagram of $g$ versus the equilibrium value coral cover. Our focus is on coral recovery when perturbed by ecological disturbances, which will have negative impacts on coral cover and parrotfish abundance. Thus, if the coral cover is perturbed to a point within the region labeled " $A$ ", the system will ultimately arrive at a coraldepleted state corresponding to high levels of algae.

In particular, hysteresis will result if we begin with low-intensity grazing followed by an increase in the grazing intensity above a critical threshold (given by $\left.g_{\text {crit }_{1}}\right)$. Once the system has surpassed this threshold and the system arrives at a stable state with high coral cover, a disturbance may result in a subsequent decline in grazing below a second critical threshold $\left(g_{\text {crit }}\right)$ and reduction in coral cover, returning the system to a degraded coral-depleted state. From Fig. $1, g_{\text {crit }_{1}}>g_{\text {crit }_{2}}$; consequently, returning to high coral state requires a grazing intensity higher than the grazing rate initially forcing the system to a coral-depleted state. Thus, preventative measures to maintain grazing rates above $g_{\text {crit }_{2}}$ in systems with high coral cover will require less drastic management than those necessary for the recov- ery of areas in which a phase shift to coral depletion has already occurred. It is also important to recognize that once there is high coral cover, further increases in grazing intensity will provide the system with greater resilience, a feature necessary for coral to withstand environmental changes.

If extremely high coral cover was realized, then the availability of food for parrotfish would decline considerably, imposing a negative food limitation on the population. We have not modeled such food limitation explicitly because such conditions virtually never arise on Caribbean reefs today; some of the healthiest reefs in the Caribbean are found in Bonaire (Kramer 2003), yet the coral tends not to exceed $56 \%$. Such reefs have a high biomass of parrotfishes (PJM, unpublished data), and therefore, we consider this to be a realistic upper limit for coral cover in our model and one that does not seem to imply food limitation. Thus, we only focus on coral dynamics that could attain a maximum level of $56 \%$. In other words, $C_{0}<0.56$, recognizing that this is an equilibrium value of coral cover $\left(C^{*}=\frac{r-d}{r}=\right.$ 0.56) so any initial coral cover below this will never reach nor exceed this point. Furthermore, we exclude the coexisting equilibrium corresponding for $C^{*}>\frac{r-d}{r}$, as it can easily be seen that this implies $M^{*}<0$ and therefore can only be approached if $M_{0}<0$ which is not biologically relevant.

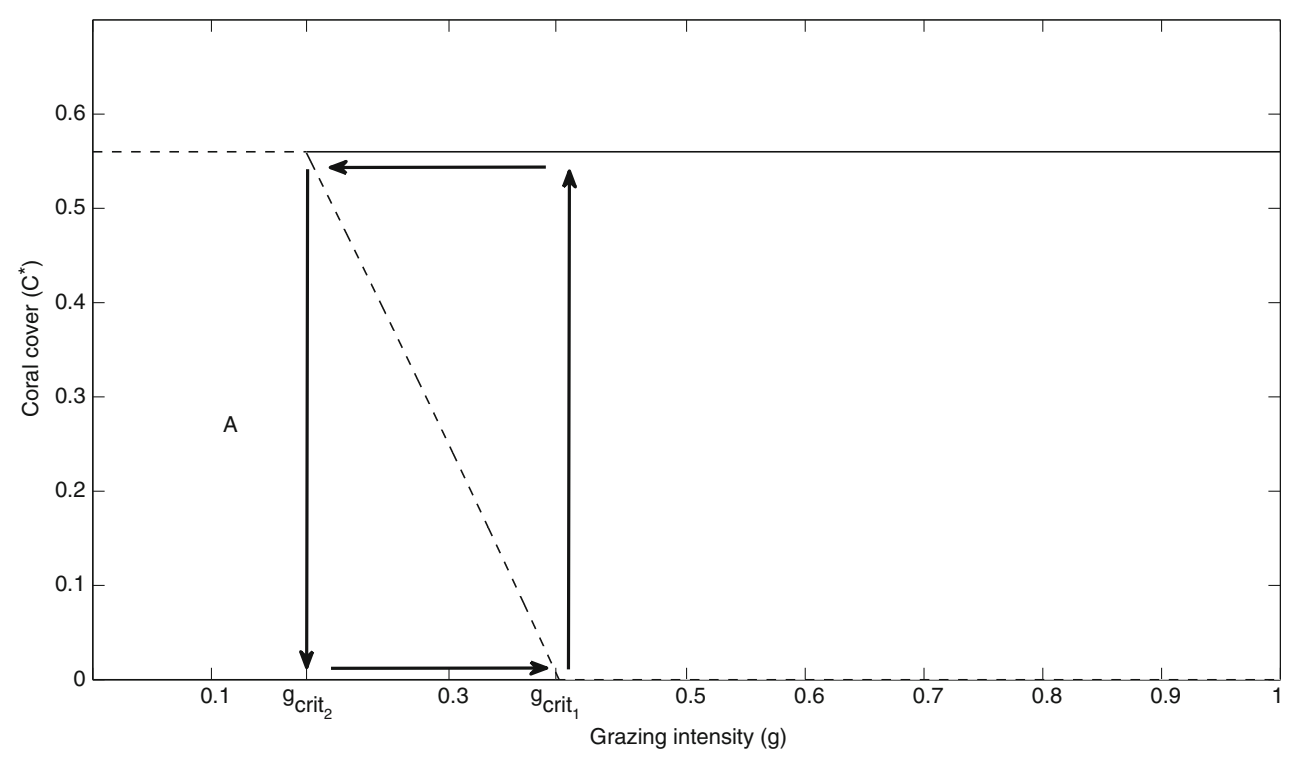

Fig. 1 Bifurcation diagram of grazing intensity $(g)$ versus the equilibrium value of coral cover $\left(C^{*}\right)$, demonstrating the possibility of hysteresis. The region labeled " $A$ " (bordered by the equilibria) represents all states in which the system will become algae-dominated without management. Solid lines represent stable equilibria, and dashed lines represent unstable equilibria, with the hysteresis loop depicted by arrows. The labeled values $g_{\text {crit }_{1}}(\approx 0.39)$ and $g_{\text {crit }_{2}}(\approx 0.18)$ identify the critical thresholds that may result in a phase shift. $g_{\text {crit }_{1}}>g_{\text {crit }_{2}}$, demonstrating the difficulty in coral recovery once the system has shifted to a macroalgal-dominated state in addition to the need for preventative measures to maintain grazing intensities greater than $g_{\text {crit }_{2}}$ 


\section{Explicit inclusion of grazer dynamics}

It has been hypothesized that excessive fishing pressure is a potential cause of hysteresis (Bellwood et al. 2004). It is our primary goal to consider explicitly the role of parrotfish abundance on grazing intensity and implement management on the system by including control on fishing pressure. We first extend the Mumby et al. model in "Original model" to account for parrotfish dynamics and treat grazing as a dynamic process:

$$
\begin{aligned}
\frac{d M}{d t} & =a M C-\frac{g(P) M}{M+T}+\gamma M T \\
\frac{d C}{d t} & =r T C-d C-a M C \\
\frac{d T}{d t} & =\frac{g(P) M}{M+T}-\gamma M T-r T C+d C \\
\frac{d P}{d t} & =s P\left(1-\frac{P}{\beta K(C)}\right)-f P
\end{aligned}
$$

where changes in parrotfish abundance, $P$, are modeled as logistic growth with an intrinsic rate of growth $s$ and a time varying carrying capacity such that $\beta$ is the maximum carrying capacity and $0<K(C) \leq 1$ is a nondimensional term that limits carrying capacity of parrotfish as a function of coral cover. Thus, habitat conditions can limit the growth of parrotfish, and different forms of this function will be explored in "Results" section. Furthermore, it is assumed that mortality resulting from fishing effort is held at a constant level $f \geq 0$. Similar to Eqs. $1-3$, this system is invariant on the region $0<M+T<1$ and $C, M, T \geq 0$.

Grazing intensity varies depending on parrotfish abundance so that grazing is now defined by as function $g(P)$. We chose the simplest function to capture increases in grazing in response to increases in parrotfish abundance. Specifically, we assume that the grazing intensity is proportional to parrotfish abundance relative to its maximum carrying capacity, or $g(P)=\frac{\alpha P}{\beta}$ where $\alpha$ is a positive constant. It is natural to let $\alpha=g_{\max }$ where $g_{\max }$ is the maximum possible grazing intensity and for simplicity it is assumed to equal one. This provides a reasonable upper bound on grazing intensity based on our previous work (Mumby et al. 2007). Thus, $g(P)=\frac{P}{\beta}$ and this formulation guarantees that the grazing intensity will arrive at a maximum only if fishing effort is eliminated $(f=0)$ and $P(t)$ has reached the maximum carrying capacity, which requires that there is no limitation from habitat (i.e., $K(C)=1$ ).
Scaling of parrotfish abundance

For simplicity, we scale parrotfish abundance to study their dynamics relative to the maximum carrying capacity, $\beta$. Specifically, we introduce the nondimensional variable $\tilde{P}$ to be parrotfish abundance relative to its maximum carrying capacity, or $\tilde{P}=\frac{P}{\beta}$. Substituting this in to Eq. 7 leaves with

$\frac{d \tilde{P}}{d t}=s \tilde{P}\left(1-\frac{\tilde{P}}{K(C)}\right)-f \tilde{P}$.

Equations 4-6 remain the same with the exception that the grazing function is now simply given by $g(P)=\tilde{P}$. Hereafter, for simplicity in notation, we set $\tilde{P}=: P$.

In the sections below, we consider two distinct scenarios of coral recovery by selecting different forms of $K(C)$ : The first assumes a coral structure that is relatively flat, presumably the result of a hurricanerelated disturbance, and the second assumes that the reef habitat is structurally complex but living coral tissue has been depleted likely a result of bleaching events. Our formulation allows us to determine when reductions in fishing effort will allow coral recovery whereas the previous model formulation lacked the associated feedbacks between parrotfish dynamics and coral cover so that the direct effect of fishing could not be evaluated. This explicit coupling of the fishing impact and the ecosystem response is the very essence of ecosystem-based management, for which relatively few examples exist (Levin and Lubchenco 2008).

\section{Results}

Habitat as primary limiting resource

In this section, we use the model introduced in "Explicit inclusion of grazer dynamics" under the assumption that parrotfish lack adequate refugia, likely a result of hurricane impacts resulting in a positive relationship between parrotfish carrying capacity and coral growth. Thus, we define the carrying capacity in Eq. 8 as

$K(C)=C$

so that there is a short-term positive response to coral recruitment. Consequently, the carrying capacity approaches a maximum value $C^{*}=0.56$ since, as described above, $C_{0}<0.56$.

Given this relationship between carrying capacity and coral cover, we determine a controllable set, or the set of all initial states within the region ultimately leading to coral depletion (region " $A$ " in Fig. 1) that can 
be returned to a state of high coral cover given some management policy. To determine this set, we assume that there is no mortality due to fishing effort, or $f=0$ (hereafter, we will refer to $f$ as fishing effort, since it is directly related to fishing mortality). Complete elimination of fishing effort is the most extreme management policy and therefore allows us to determine the maximum number of initial states in " $\mathrm{A}$ " that allow for coral recovery in response to changes in grazing levels. We produced a grid of equally spaced points in the region labeled " $A$ " in Fig. 1 that correspond to initial values of coral cover and grazing intensity (see Fig. 2a). By simulating the updated model (Eqs. 4-7) from these different grid points, we are able to identify which points are capable of going through a phase shift from a coral-depleted (with much macroalgae) to a state providing a stable balance between algal turfs and coral (given by the equilibrium value $\left(M^{*}, C^{*}, T^{*}\right)=$ $\left.\left(0, \frac{r-d}{r}, 1-C^{*}\right)\right)$ in a finite time of $t$ years. We call this our controllable set.

Figure $2 \mathrm{~b}-\mathrm{d}$ displays the results for a control period of 5 years under different initial states. We recognize
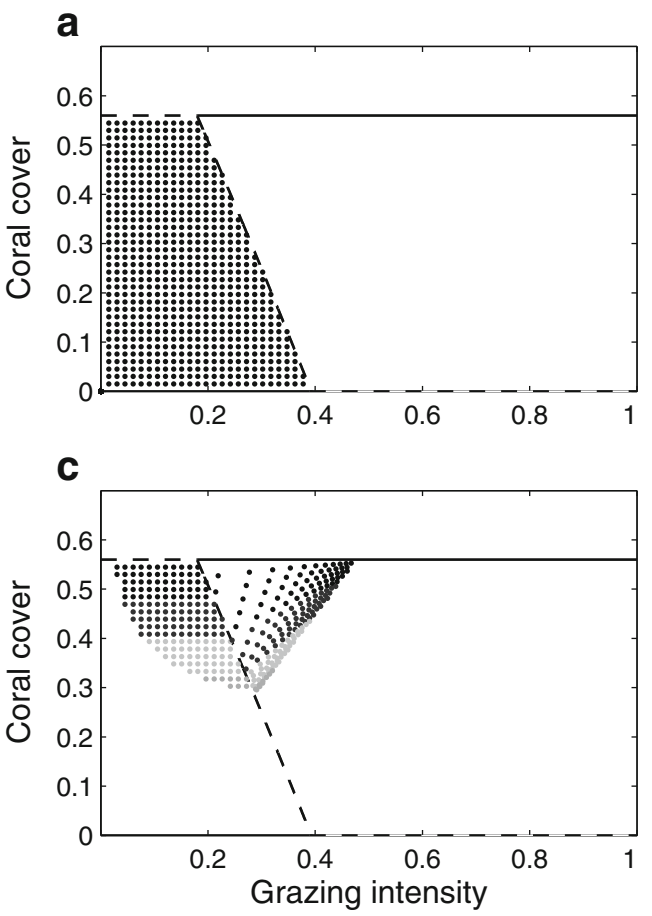

Fig. 2 Simulation of points in region " $A$ " from Fig. 1 for 5 years. Beginning with an initial grid of evenly spaced points in "A" which determine the initial level of coral cover and grazing intensity (a), we simulate our model for 5 years, assuming $f=0$. It is first assumed that the initial macroalgal cover, $M_{0}$, is equal to zero (b). Second, it is assumed that $M_{0}$ is at its nontrivial equilibrium corresponding to $C_{0}(\mathbf{c})$. Finally, $M_{0}=1-C_{0}$, where that the initial coral and macroalgal cover will play substantial roles in determining the controllable regions. However, providing a complete evaluation of all states is computationally difficult, so we consider three distinct scenarios for initial conditions of algal turfs, macroalgae, and coral cover and numerically determine their dynamics. Our analysis is then displayed as projections of sets of initial states that allow for coral recovery as well as their corresponding ending location onto Fig. 1. This allows us to determine the states ultimately leading to coral depletion in the original model (Eqs. 13) that may regain coral cover in the absence of fishing effort for the updated model (Eqs. 4-7).

In particular, Fig. $2 \mathrm{~b}$ represents the "best" possible initial conditions; for each initial coral cover and grazing intensity, there is no macroalgae allowing for greater coral recruitment giving rise to better habitat conditions. This corresponds to an environmental disturbance that eliminated macroalgal cover and reduced coral cover to corresponding starting value of the gridpoint in Fig. 2b. Under these circumstances, it is evident that in nearly every initial state, coral recovery

b

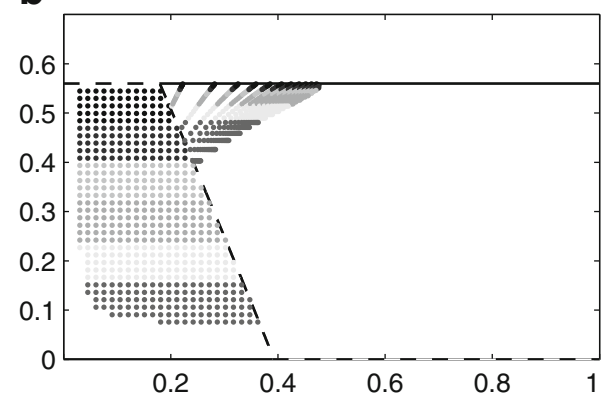

d

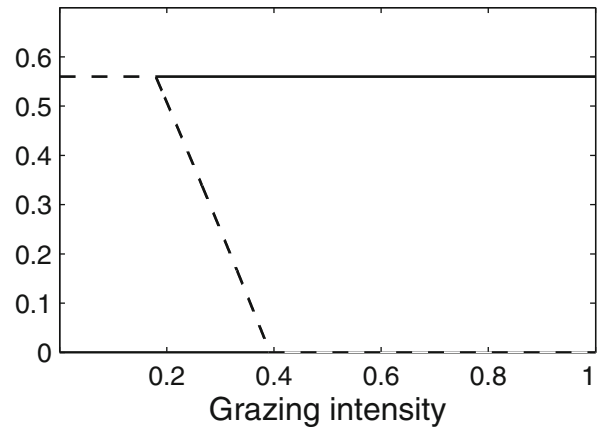

$C_{0}$ is the initial coral cover (d). Additionally, the initial grazing intensity corresponds to the initial parrotfish density by our construction since $g(P)=P$. In $\mathbf{b}-\mathbf{d}$, the points within region " $A$ " (labeled in Fig. 1) indicate the controllable set and their corresponding ending location is outside of this region. The points are shaded to emphasize their specific starting and end location. See Online Resource 2 for a sensitivity analysis of these results 
is promoted. Figure $2 \mathrm{c}$ represents a system in which the coral cover been affected resulting in severe habitat degradation and the macroalgal cover is at the equilibrium corresponding to the coexistence of coral and macroalgae for the given $C_{0}$, providing an intermediate level of macroalgal cover. Finally, Fig. 2d demonstrates the least favorable circumstances in which the initial cover of the seabed is completely dominated by coral and macroalgae (i.e., $C_{0}+M_{0}=1$ ), initially preventing coral from recruiting to algal turfs. Here, the initial states that will drive the system to a high coral cover is limited even with a complete elimination of fishing effort.

Figure 2 demonstrates two primary results: The controllable set heavily depends on the initial values of coral and macroalgal cover, and analytically finding a controllable region is a challenging task. As a result of this difficulty, our analysis thus far has been primarily numerical. Furthermore, Fig. 2 assumes that for a time period of 5 years, the complete elimination of fishing effort is feasible. There is, however, a high economic impact on fisheries that may require a different strategy for optimal management leading us to examine conditions under which different fishing effort levels may still allow the original system to undergo hysteresis (see Fig. 3a). This provides insight into conditions under which it is vital to reduce or completely eliminate fishing effort based on the initial severity of habitat degradation (independent of initial grazing intensities).

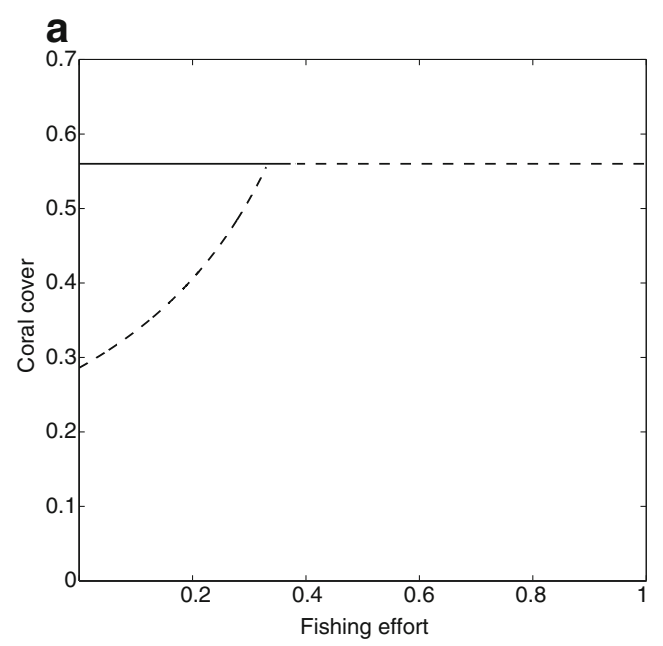

Fig. 3 Bifurcation diagram of the equilibrium value of coral cover $(C)$ versus the level of fishing effort $(f)$. Solid lines are stable equilibria, and dashed lines are unstable equilibria. With habitat as the primary limiting factor, sufficiently low levels of fishing effort will allow for coral recovery for some initial states, but the lower the effort, the greater the number of initial states promoting coral recovery (a). In $\mathbf{b}$, with food availability as the
Clearly, if fishing effort exceeds the population growth rate of parrotfish, there is no chance for coral recovery. Additionally, for sufficiently low levels of fishing effort, there are two stable states (coral-depleted and coraldominated), and therefore, it is evident that recovery will depend on the initial state (as demonstrated in Fig. 2 for the case $f=0$ ). It is clear that with a decrease in fishing pressure, coral dominance becomes more viable, further demonstrating the importance of fishing regulation in coral recovery and preservation.

Food availability as primary limiting resource

We now consider reefs following bleaching events or hurricanes that do not damage the reef structure itself but instead deplete coral abundance, freeing up space for algal colonization. Thus, sufficient refugia remains and parrotfish respond positively to increases in food availability. Equivalently, further increases in coral cover have a negative impact on parrotfish carrying capacity, a result of the assumption that $M+T=1-$ $C$, which implies increasing coral cover reduces food supply and therefore carrying capacity for parrotfish under a food-limitation scenario. We represent this scenario of coral recovery by defining the $K(C)$ in Eq. 8 by

$K(C)=1-z C$

b

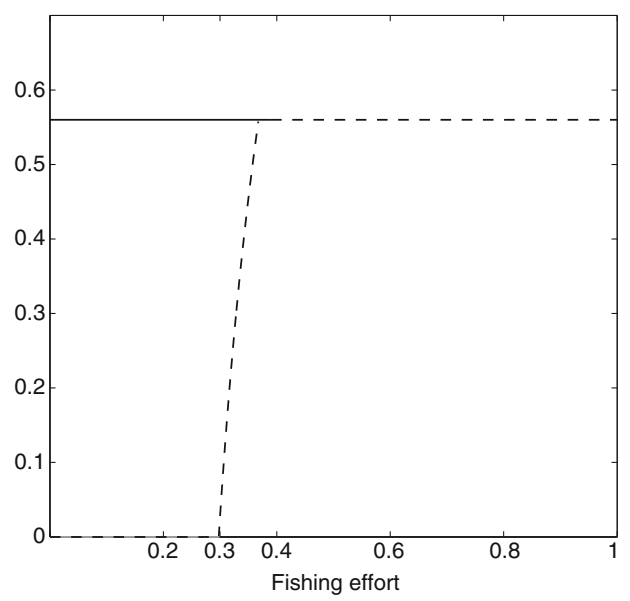

primary limiting parrotfish resource, effort levels below $f_{\text {crit }} \approx$ 0.3 will guarantee coral recovery. For intermediate values of effort, there is potential for coral recovery depending on the initial states, and macroalgae will dominate the system if $f$ is raised to levels beyond $f_{\text {crit }_{2}}(\approx 0.37)$. In both figures, if fishing effort level exceeds the growth rate of parrotfish $(s=0.49)$, as expected there is no chance of coral recovery 
a

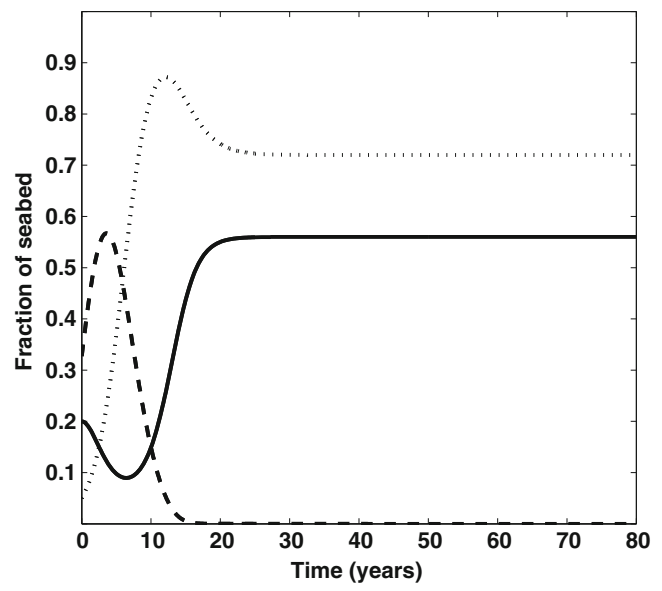

Fig. 4 Time integrated dynamics of coral (solid line) and macroalgal (dashed line) cover and number of parrotfish relative to the maximum carrying capacity (dotted line). Initial grazing level is $0.05, C_{0}=0.2$, and $M_{0}$ is at its equilibrium value with fishing effort levels of $f=0$ (a) and $f=0.25$ (b). Increased

where $0 \leq z<1$ (we fix this value but as shown in Online Resource 2 , the dynamics are qualitatively similar for all values of $z$ ).

We first highlight the effects of fishing effort on coral recovery. Figure $3 b$ shows the bifurcation diagram of the equilibrium value of coral cover versus the level of fishing effort. Again, effort levels greater than the intrinsic growth rate of parrotfish will always lead to algal dominance. Contrary to the previous section, however, we observe that there is greater resilience to fishing effort when parrotfish are primarily limited by food abundance. Specifically, when fishing effort levels are below a value of approximately $f_{\text {crit }}=0.3$, the only stable state corresponds to high coral cover indicating that this state is stable for all initial conditions. Therefore, we omit numerically determined controllable sets because in this scenario all initial states allow for recovery given that $f=0$.

We now consider the dynamics of coral and macroalgae in response to different levels of fishing effort. In Fig. 4a, we choose $f=0$, and in Fig. $4 \mathrm{~b}$, we increase the level of fishing effort to $f=0.25$. For both effort levels, coral cover is negatively impacted by macroalgal cover after a short time lag-a consequence of the ability of macroalgae to recruit to and overgrow coral. However, this results in an increased food availability allowing parrotfish recovery and a subsequent increase in $g(P)$. Thus, macroalgae eventually declines and coral is able to recover. Although Fig. 4a, b demonstrates coral recovery, it is important to recognize the difference in recovery time related to the corresponding effort level.

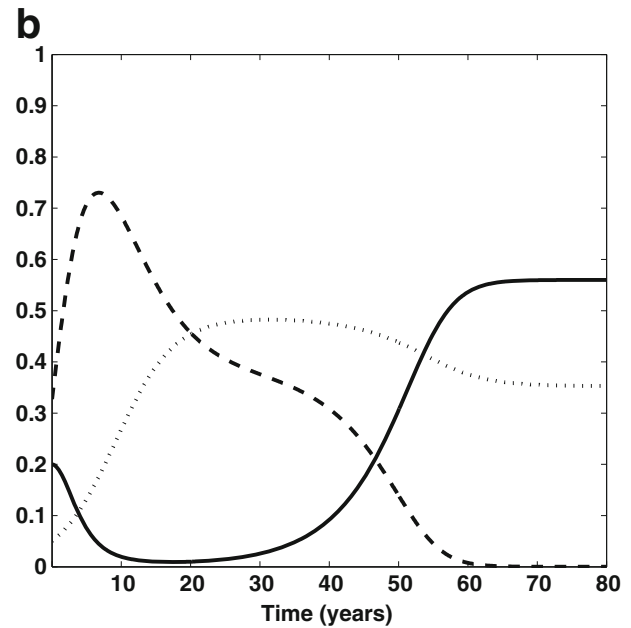

fishing effort requires a substantially longer period of time for coral recovery demonstrating the essentiality of reduced effort or MPA implementation. Both figures display an initial drop in coral cover before they begin to recover as a result of the time required for parrotfish to adequately recover

While complete coral recovery takes approximately 20 years with no fishing effort, it takes more than 60 years when this level is increased to 0.25 . Therefore, short-term recovery is more possible with drastic management, such as through the implementation of marine protected areas (MPA) or a cessation to herbivore exploitation.

\section{Discussion}

Ecosystem resilience is important for maintaining a favorable state in the presence of environmental and human impacts. As reviewed by Folke et al. (2004), reductions in resilience from humans (because of pollution, over-harvesting, spraying pesticides, etc.) may result in a regime or phase shift to a degraded or less desirable state. However, subsequent changes in human activities aimed at regaining resilience (e.g., through fishing reductions) may again lead to a phase shift to the more desirable state and allow for ecosystem recovery. While hysteresis has been observed both empirically and theoretically (e.g., Ludwig et al. 1978; Scheffer and Carpenter 2003), the incorporation of resilience theory into practical management is in its infancy yet will almost certainly be necessary if countries are to fulfill their obligations to undertake ecosystem-based management and fisheries (Ruckelshaus et al. 2008). Here, we extended an earlier model of a coral reef ecosystems that had grazing intensity of algae at an imposed level 
to explicitly consider the impacts of fishing on grazing intensity which in turn effects ecosystem's resilience. Moreover, this particular formulation allows us to develop two realistic scenarios for the implementation of such ecosystem-based management: one in which the system has recently experienced major catastrophe leaving little coral structure and a second in which the reef structure is fully developed but subjected to the impacts of disease or pollution such as coral bleaching. We learn that the management responses differ in each scenario.

After massive catastrophe, such as may occur after a hurricane impact or ship grounding in which much reef structure is lost, the system has relatively limited resilience and alternate stable states are feasible across a range of fairly low fishing intensities. Under these circumstances, restorative interventions that add coral cover and build vertical relief may help avoid entrainment to a coral-depleted state and encourage recovery. Such restoration is only ever practical at small scales (Edwards 2008), yet this might be feasible for treating the sorts of localized disturbance that have profound effects on reef structure.

Our theoretical model suggests that reefs with intact structure are more resilient, exhibiting a relatively wide range of fishing intensities in which coral recovery is promoted. At low fishing intensities, coral populations should have the potential to exhibit periods of recovery between successive disturbances, helped in part by the positive numeric response of parrotfishes to increased food availability after coral mortality. However, as fishing effort increases toward a critical bifurcation threshold, the time needed for recovery increases dramatically (up to threefold). With the widespread increase in climatic disturbances on coral reefs (HoeghGuldberg et al. 2007), managers face the challenge of maintaining ecosystem services across the seascape. Efforts to enhance the rate of coral recovery are central to meeting this challenge, and therefore, reducing the fishing of herbivores may have a nonlinear benefit on ecosystem recovery.

We recognize that our model is one step in understanding coral reef ecosystems. There are other factors that we have not explicitly included that clearly would affect the dynamics, but we argue that these effects would modify the results while not changing the basic import of our conclusions. For example, we neglect sedimentation which causes degradation of coral reefs; accounting for this could result in changes to the settlement space available for coral and algae (Rogers 1990). Furthermore, we assume that corals are overgrown by macroalgae at a constant rate. However, nutrification may promote faster colonization of available seabed by algal turfs and macroalgae which in turn would inhibit the settlement of coral (Costa et al. 2000). Moreover, we implement fishing effort as a constant rate of parrotfish mortality which neglects variations in effort resulting from seasonality and fishery profits. Thus, a possible extension of this model is to apply a more direct economic analysis that considers fishery response to profits.

Although we have considered two distinct scenarios of coral recovery, we appreciate that these may occur sequentially, with habitat limitation (post coral catastrophe) eventually being followed by food limitation if the reef recovers. We will examine the time scales involved in a later study but note that each scenario currently represents a different spatial scale; habitat limitation is currently likely to be a small-scale and patchy phenomenon whereas food limitation applies more widely over the seascape. Habitat limitation is often caused by intense mechanical disturbance to reef structure arising from hurricanes or ship groundings, which is often extremely patchy at local scales (Done 1992; Bythell et al. 2000). Food limitation, on the other hand, often occurs when coral bleaching leads to mortality of the coral without affecting the habitat structure (at least in the short term). Bleaching events are usually spatially extensive, extending over hundreds to thousands of kilometers (Wilkinson 1998). Of course, with increased climatic stress and likely reductions in pelagic dispersal (O'Connor et al. 2007), good quality habitat is set to become increasingly fragmented in future, and our scenario of habitat limitation will become increasingly relevant. Given that this scenario has lower natural levels of resilience, every effort needs to be made to limit the loss of reef structure through improved reef management and reduced emissions of greenhouse gases.

More generally, we have provided a concrete example demonstrating how ideas from hysteresis and resilience can play an important role in understanding management options. In particular, we identify those states from which recovery is possible within the range of management options possible within the system, namely options for altering fishing effort. Understanding this important feature of controllability is an essential first step in preserving systems, even before economic analyses are applied. This aspect of determining states from which recovery is possible will apply much more broadly than the coral reef system; however, by illustrating the analysis in this particular system, we demonstrate the feasibility and importance of such an analysis. 
Acknowledgements This work was partially supported by the National Science Foundation under the Grants \#DMS0636297 and \#EF-0742674, U.S. EPA Science to Achieve Results (R832223), and the Natural Environment Research Council. We also thank two anonymous reviewers for their helpful comments.

Open Access This article is distributed under the terms of the Creative Commons Attribution Noncommercial License which permits any noncommercial use, distribution, and reproduction in any medium, provided the original author(s) and source are credited.

\section{References}

Bellwood DR, Hughes TP, Folke C, Nystrom M (2004) Confronting the coral reef crisis. Nature 429:827-833

Box SJ, Mumby PJ (2007) The effect of macroalgal competition on the growth and survival of juvenile Caribbean corals. Mar Ecol Prog Ser 342:139-149

Bythell JC, Gladfelter EH, Bythell M (1993) Chronic and catastrophic natural mortality of three common Caribbean reef corals. Coral Reefs 12:143-152

Bythell JC, Hillis-Starr ZM, Rogers CS (2000) Local variability but landscape stability in coral reef communities following repeated hurricane impacts. Mar Ecol Prog Ser 204: 93-100

Carpenter RC, Edmunds PJ (2006) Local and regional scale recovery of Diadema promotes recruitment of scleractinian corals. Ecol Lett 9:271-280

Connell JH (1997) Disturbance and recovery of coral assemblages. Coral Reefs 16:S101-S113

Costa Jr OS, Leão Z, Nimmo M, Attrill MJ (2000) Nutrification impacts on reefs from northern Bahia, Brazil. Hydrobiologia 440:307-315

Done TJ (1992) Effects of tropical cyclone waves on ecological and geomorphological structures on the Great Barrier Reef. Cont Shelf Res 12:859-872

Done TJ, Ogden JC, Wiebe WJ, Rosen BR (1996) Biodiversity and ecosystem function of coral reefs. In: Mooney HA, Cushman JH, Medina E, Sala OE, Schulze ED (eds) Functional roles of biodiversity: a global perspective. Wiley, New York, pp 393-429

Edwards AJ (2008) What role for reef restoration in the face of climate change? Reef Encounter 36:12-14

Floeter RJ, Behrens MD, Ferreira CEL, Paddack MJ, Horn MH (2005) Geographical gradients of marine herbivorous fishes: patterns and processes. Mar Biol 147:1435-1447

Folke C et al (2004) Regime shifts, resilience, and biodiversity in ecosystem management. Annu Rev Ecol Evol Syst 35:557581

Hoegh-Guldberg O et al (2007) Coral reefs under rapid climate change and ocean acidification. Science 318:1737-1742
Holling CS (1973) Resilience and stability of ecological systems. Annu Rev Ecol Syst 4:1-23

Hughes TP (1994) Catastrophes, phase shifts, and large-scale degradation of a Caribbean coral reef. Science 265:15471551

Jompa J, McCook LJ (2002) Effects of competition and herbivory on interactions between a hard coral and a brown alga. J Exp Mar Biol Ecol 271:25-39

Knowlton N (1992) Thresholds and multiple stable states in coral reef community dynamics. Am Zool 32:674-682

Kramer PA (2003) Synthesis of coral reef health indicators for the Western Atlantic: results of the AGRRA program (1997-2000). Atoll Res Bull 496:1-58

Levin SA, Lubchenco J (2008) Resilience, robustness, and marine ecosystem-based management. Bioscience 58:27-32

Lirman D (2001) Competition between macroalgae and corals: effects of herbivore exclusion and increased algal biomass on coral survivorship and growth. Coral Reefs 19:392-399

Ludwig D, Jones DD, Holling CS (1978) Qualitative analysis of insect outbreak systems: spruce-budworm and forest. J Anim Ecol 47:315-32

Moberg F, Folke C (1999) Ecological goods and services of coral reef ecosystems. Ecol Econ 29:215-233

Mumby PJ, Foster NL, Glynn Fahy EA (2005) Patch dynamics of coral reef macroalgae under chronic and acute disturbance. Coral Reefs 24:681-692

Mumby PJ et al (2006) Fishing, trophic cascades, and the process of grazing on coral reefs. Science 311:98-101

Mumby PJ, Hastings A, Edwards H (2007) Thresholds and the resilience of Caribbean coral reefs. Nature 450:98-101

O'Connor MI et al (2007) Temperature control of larval dispersal and the implications for marine ecology, evolution, and conservation. Proc Natl Acad Sci U S A 104:1266-1271

Rogers CS (1990) Responses of coral reefs and reef organisms to sedimentation. Mar Ecol Prog Ser 62:185-202

Ruckelshaus M, Klinger T, Knowlton N, DeMaster DP (2008) Marine ecosystem-based management in practice: scientific and governance challenges. Bioscience 58:53-63

Scheffer M, Carpenter SR (2003) Catastrophic regime shifts in ecosystems: linking theory to observation. Trends Ecol Evol 18:648-656

Scheffer M, Carpenter S, Foley JA, Folke C, Walker B (2001) Catastrophic shifts in ecosystems. Nature 413:591-596

Tolimieri N (1998) Effects of substrata, resident conspecifics and damselfish on the settlement and recruitment of the stoplight parrotfish, Sparisoma viride. Environ Biol Fisches 53:393404

von de Koppel J, Rietkerk M, Weissing FJ (1997) Catastrophic vegetation shifts and soil degradation in terrestrial grazing systems. Trends Ecol Evol 12:353-356

Williams ID, Polunin NVC (2000) Large-scale associations between macroalgal cover and grazer biomass on mid-depth reefs in the Caribbean. Coral Reefs 19:358-366

Wilkinson C (1998) Status of coral reefs of the world: 1998. Australian Institute of Marine Science, Townsville 